\title{
Chondral print on humeral head: an indirect sign of long head biceps tendon instability
}

\author{
Alessandro Castagna $\cdot$ Elyazid Mouhsine $\cdot$ Marco Conti $\cdot$ Enzo Vinci $\cdot$ \\ Mario Borroni · Antonio Giardella $\cdot$ Raffaele Garofalo
}

Received: 11 July 2006/ Accepted: 31 August 2006/ Published online: 10 November 2006

(C) Springer-Verlag 2006

\begin{abstract}
Long head biceps (LHB) tendon pathologies are becoming increasingly recognized causes of shoulder pain in the published literature. Instability of LHB presenting as dislocation or subluxation has been recently recognized as a possible cause of disabling pain or discomfort of the shoulder. A clinical diagnosis of LHB instability is very difficult and often confounding because of association with other shoulder pathologies. However, an early diagnosis of LHB instability is important in order to prevent the evolution of lesions of the biceps pulley until an internal anterosuperior impingement of the shoulder (ASI) and subscapular tear occur. The advent of arthroscopy contributed to enhance understandings. The goal of this article is to describe an arthroscopic sign, the chondral print on the humeral head, associated with a LHB instability, that when present can be very useful to help the surgeon to make the diagnosis of unstable LHB tendon.
\end{abstract}

Keywords Long head biceps tendon - Instability ·

Shoulder · Arthroscopy · Pulley

\section{Introduction}

Pathological conditions of long head of biceps (LHB) tendon have been well recognized in the published literature as a cause of painful shoulder $[3,6]$. LHB lesions

A. Castagna - E. Mouhsine - M. Conti - E. Vinci .

M. Borroni · A. Giardella · R. Garofalo

Shoulder Unit Service, Humanitas Institute, Milan, Italy

E. Mouhsine $(\varangle)$

Ch. Du Pré-de-Jean, 1174 Montherod, Switzerland

e-mail: Elyazid.mouhsine@chuv.ch requiring treatment include SLAP lesion, tendinitis, partial-thickness tears and instability. Instability of LHB presenting as dislocation or subluxation [12] associated more or less to rotator cuff tear [8] and causing eventually an anterosuperior impingement syndrome (ASI) [2] may be responsible for disabling pain or discomfort of the shoulder. The clinical diagnosis of LHB instability is difficult and confounding because the association with other shoulder pathologies is very frequent. The advent and progress of arthroscopy has contributed to enhance understanding and in consequence the treatment of this pathology. The lesion of pulley system has been identified as the "primum movens" in the LHB instability cascade [5]. Identifying a complete LHB tendon dislocation associated with a major lesion of pulley system is, in general, not difficult, and its treatment can be straightforward.

On the other hand, also under arthroscopic view, a chronic subluxation of LHB can be missed, because the minimal dynamic medial displacement of LHB tendon can be not easy to detect. In this condition, the pulley system lesion can be minimal and difficult to identify, and also other indirect signs such as fraying of superior edge of subscapular can be lacking. In this situation the instability of LHB is obscured, and also if the surgeon tests with a probe or a shaver the tendon to demonstrate that it is instable, it is very difficult to confirm it. If this pathology is missed, and so left untreated, the patient will continue to experience shoulder discomfort and pain after operation.

The purpose of this paper is to describe an arthroscopic sign, the chondral print on the humeral head, as a useful marker of the unstable LHB. This finding when present can help the surgeon to establish a diagnosis of unstable LHB tendon. 


\section{Case report}

In the period between January 2003 and March 2005 the senior author performed 1,300 shoulder arthroscopies. All the procedures were in lateral decubitus position under interscalenic block anesthesia. Balanced suspensions of 5-10 $\mathrm{lb}$ are used with the arm in $20^{\circ}-30^{\circ}$ of abduction and $20^{\circ}$ of forward flexion using a dedicated traction system (Arthrex, Naples FL, USA). A $30^{\circ}$ arthroscope was introduced at first through a standard posterior portal. An anterior-superior (AS) portal was established with an inside-out technique. With a probe introduced through the AS portal a systematic diagnostic arthroscopy of the glenohumeral joint was carried-out. Visualization of the LHB tendon and pulley is best performed through the posterior viewing portal and the anterior probe can be used to test the stability of LHB tendon. The patients with an unstable LHB tendon were prospectively recorded. There were 550 patients with a diagnosis of unstable LHB tendon. Of these, 400 patients were excluded because of a dislocated LHB tendon associated with a large rotator cuff tear. The remaining 50 patients were included in this study. There were 98 men and 52 women with a mean age of 48.3 years (range 20-69 years). Of them, 70 patients had a history of trauma, whereas 80 had none. The review of the preoperative clinical charts revealed the presence of a positive palm-up test in $120(80 \%)$ patients and a positive O'Brien test in 122 (82\%) patients. In 115 (77\%) patients the palpation of region of LHB at the level of anterior region of shoulder was painful. Only in four $(2 \%)$ patients none of the previous three tests was positive. In $18(12 \%)$ patients a stiff shoulder was present at preoperative clinical examination.

Of these 150 unstable LHB tendons, at arthroscopic examination, $92(61 \%)$ were judged as dislocated and $58(39 \%)$ as subluxable. In case of a dislocated LHB tendon a lesion of pulley system was evident that was classified retrospectively, reviewing recorded videos, according to criteria of Habermeyer [5] as type II (20 cases), type III (32 cases) and type IV (40 cases). In all these 92 cases a chondral print lesion at the level of humeral head was found. Among the 58 cases in which the LHB was judged as subluxable, it was possible to find clear signs of pulley lesion in $49(84 \%)$ patients, in particular, in 28 cases was classified as type I, 14 patients as type II and in the other 7 patients as type III according to Habermeyer criteria [5]. In 52 (89\%) of these 58 patients it was possible to evidence a chondral print at the level of humeral head. Interestingly in all 9 $(15 \%)$ patients in whom the pulley system lesions were not very clear, the presence of a chondral print at the level of humeral head get us the diagnosis of unstable LHB tendon (Fig. 1). In these cases, the presence of some localized inflammation of the synovial tissue at the level of rotator interval and at the level of the superior edge of subscapularis tendon without a real fraying was noted (Figs. 2, 3). Moreover, other indirect signs of LHB tendon sufference such as a thickening, flattening and partial tear or fraying at the level of intraarticular course were found in four of these nine cases (Fig. 4).

The treatment performed for LHB tendon instability was a simple tenotomy in 86 patients and soft tissue tenodesis [1] in the other 64 patients, depending on age, associated signs of LHB tendon sufferance and associated tendon cuff tear.

\section{Discussion}

The instability of LHB tendon is a well-recognized cause of shoulder discomfort or pain. Walch [12] noted that a subluxation and dislocation of the biceps occurred in $16 \%$ of treated rotator cuff tear. Really, clinical diagnosis of LHB instability is difficult and confounding. In our report as in other [5] different signs of LHB sufferance such as palm-up test and O'Brien test are positive, but none of them is really specific to give a diagnosis of LHB instability. The advent of arthroscopy has contributed to improve the diagnosis of this pathology that seems to be more and more frequent. Some authors $[4,9]$ also suggest that

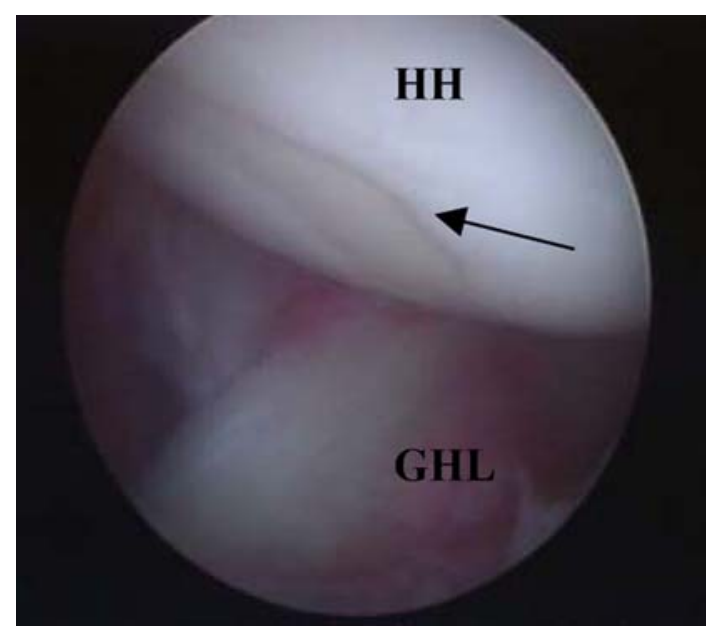

Fig. 1 Arthroscopic view from a posterior portal of a right shoulder using a $30^{\circ}$ arthroscope that shows a chondral print lesion (dark arrow) on anterior part of humeral head $(\mathrm{HH})$. Note a thickened, inflammed middle glenohumeral ligament $(G H L)$. Note that, unless otherwise noted, all figures are oriented from a lateral decubitus perspective 


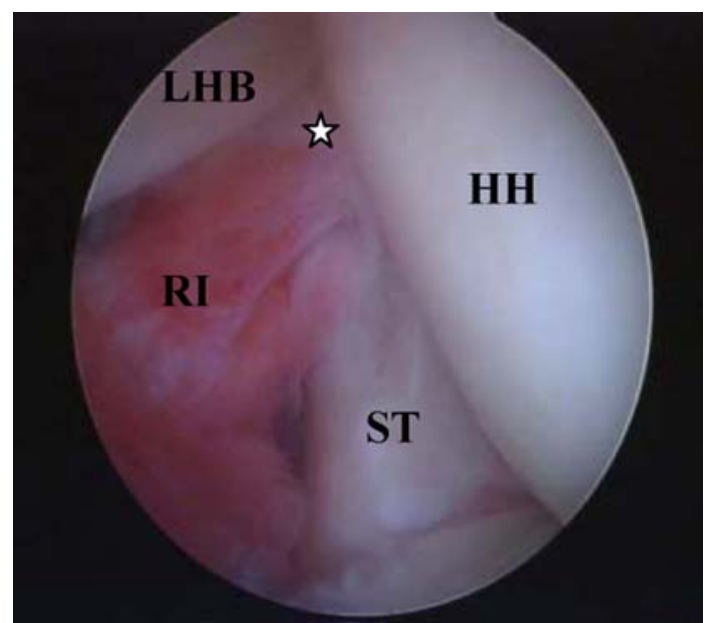

Fig. 2 The same case of the previous figure, with arthroscopic view from a posterior portal with the arm in internal rotation, showing an inflamed rotator interval $(R I)$, an intact superior edge of subscapularis tendon $(S T)$. Note the intact medial sling of the pulley system (star). $H H$ humeral head, $L H B$ long head biceps tendon

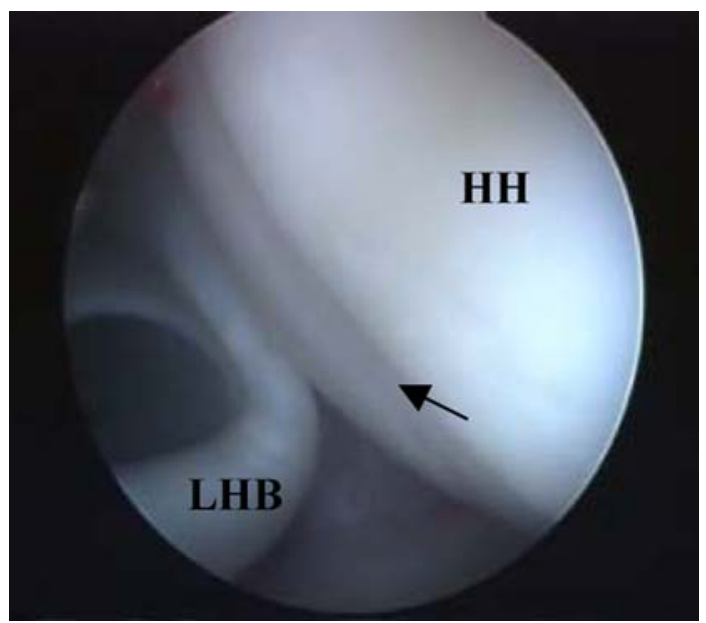

Fig. 3 The same case of the previous figure, with arthroscopic view from a posterior portal with the arm in neutral rotation, showing as the long head biceps tendon $(L H B)$ tendon is medially unstable when tested with an arthroscopic cannula. Note as the subluxable LHB tendon is the responsible of the chondral print on the humeral head (arrow). $\mathrm{HH}$ humeral head

dislocations of the LHB tendon may occur without associated abnormalities in the rotator cuff. The lesion of pulley system, in particular, has been well investigated and recognized as the primum movens of the LHB tendon instability $[10,11]$. The pulley lesion, in particular, can be caused by trauma or degenerative changes $[5,7]$. Once the reflection pulley is torn, the LHB becomes unstable in its intraarticular course leading to medial subluxation and alteration of LHB itself. Arthroscopic diagnosis in case of the LHB

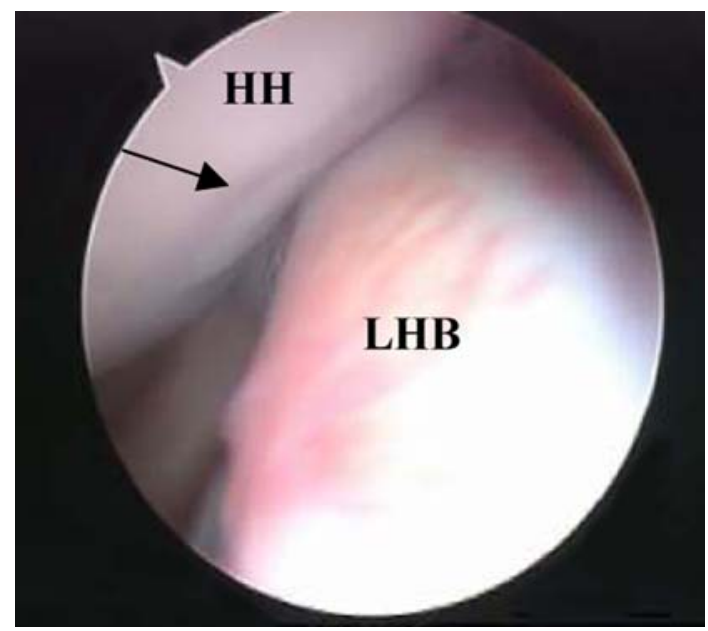

Fig. 4 Arthroscopic view of a left shoulder. Note a very large, thickened long head biceps tendon $(L H B)$ that was medially unstable. Note also the little chondral print (arrow) on humeral head $(H H)$

tendon dislocation is easily established. However, diagnosis of LHB tendon subluxation cannot be easy to do. Sometimes, lesion of pulley system can be difficult to evidence, especially at the initial stages of rupture at the level of reflection region. In consequence, also under arthroscopy, subluxation of LHB tendon can be missed because of the minimal medial displacement of tendon that it is not easy to detect and superior tendon edge of the subscapularis tendon that can appear intact [12]. A recent anatomical study [4] has shown that fibers from the subscapularis tendon divide and form an annular sling around the LHB tendon, so contributing to stability of tendon itself. This anatomical description supports the concept that in biceps instability, the deep fibers from the subscapularis tendon would tend to avulse, allowing for dislocation of the tendon in the joint. However, this partial rupture of deep fibers of subscapularis tendon sometimes may be not visible, overall at early stages of pulley system rupture. In the present report, in the nine patients in whom the pulley system lesions were not very clear and the presence of a chondral print at the level of humeral head get us the diagnosis of unstable LHB tendon, the presence of a some localized inflammation at the level of superior edge of subscapularis tendon without a true fraying was noted.

We think that this observation may be very important. In fact, the pathology of the LHB tendon instability seems to be a progressive pathology coming from a subluxable to a dislocate LHB tendon, just to lead to a partial articular-sided tear of fibers of subscapularis tendon [12] and supraspinatus tendon. Moreover, the medially displaced LHB results in increasing anterior 
translation of humeral head that can become more important in presence of an associated tear of superior edge of the subscapularis tendon allowing a further anterior-superior translation of the humeral head resulting in ASI [2].

In patients presenting with positive test for LHB pathology at preoperative clinical examination, the presence of chondral print should be looked for during an arthroscopic examination, because this sign, when present can be an useful tool that contribute to make a diagnosis of unstable LHB when the diagnosis is not yet straightforward.

\section{References}

1. Castagna A, Conti M, Mouhsine E, Bungaro P, Garofalo R (2005) Arthroscopic biceps tendon tenodesis: the anchorage technical note. Knee Surg Sports Traumatol Arthrosc1-5 (Epub)

2. Gerber C, Sebesta A (2000) Impingement of the deep surface of the subscapularis tendon and reflection pulley on the anterosuperior glenoid rim a preliminary report. J Shoulder Elbow Surg 9:483-490

3. Gill TJ, McIrvin E, Mair SD, Hawkins RJ (2001) Results of biceps tenotomy for treatment of pathology of the long head of biceps brachii. J Shoulder Elbow Surg 10:247-249
4. Gleason PD, Beall DP, Sanders TG, Bond JL, Ly JQ, Holland LL, Pasque CB (2006) The transverse humeral ligament: a separate anatomical structure or a continuation of the osseous attachment of the rotator cuff? Am J Sports Med 34:72-77

5. Habermeyer P, Magosch P, Pritsch M, Scheibel MT, Lichtenberg S (2004) Anterosuperior impingement of the shoulder as a result of pulley lesions: a prospective artroscopic study. J Shoulder Elbow Surg 13: 5-12

6. Hitchcock JJ, Bechtol CO (1948) Painful shoulder: observations on the role of the tendon of the long head of the biceps brachii in its causation. J Bone Joint Surg Am 30:263273

7. Le Huec JC, Liquois F, Schaeverbecke T, Zipoli B, Chauveaux D, Le Rebeller A (1996) Traumatic tear of the rotator interval. J Shoulder Elbow Surg 5:41-46

8. O'Donoghue DH Subluxing biceps tendon in the athlete (1982) Clin Orthop 26-29

9. Railhac JJ, Poey C, Maquin P et al (1991) Luxations traumatiques du tendon du long biceps. Encycl Med Chir Instantanes Medicaux

10. Sakurai G, Ozaki J, Tomita Y, Kondo T, Tamai S (1998) Incomplete tears of the subscapularis tendon associated with tears of the supraspinatus tendon: cadaveric and clinical studies. J Shoulder Elbow Surg 7:510-515

11. Slatis P, Aalto K (1979) Medial dislocation of the tendon of the long head of the biceps brachii. Acta Orthop Scand 1:7377

12. Walch G, Nove-Josserand L, Boileau P, Levigne C (1998) Subluxatoins and dislocations of the tendon of the long head of the biceps. J Shoulder Elbow Surg 7:100-108 